\title{
FORMATION OF SOLITONS IN ATOMIC BOSE-EINSTEIN CONDENSATES BY DARK-STATE ADIABATIC PASSAGE *
}

\author{
G. Juzeliūnas $^{\text {a }}$, J. Ruseckas ${ }^{\text {a }}$, P. Öhberg ${ }^{\mathrm{b}}$, and M. Fleischhauer ${ }^{\mathrm{c}}$ \\ ${ }^{a}$ Institute of Theoretical Physics and Astronomy of Vilnius University, A. Goštauto 12, LT-01108 Vilnius, Lithuania \\ E-mail: gj@itpa.lt \\ ${ }^{\mathrm{b}}$ Department of Physics, School of Engineering and Physical Sciences, Heriot-Watt University, Edinburgh EH14 4AS, UK \\ ${ }^{\mathrm{c}}$ Fachbereich Physik, Technische Universität Kaiserslautern, D-67663 Kaiserslautern, Germany
}

Received 27 June 2007; revised 20 August 2007

\begin{abstract}
We propose a new method of creating solitons in elongated Bose-Einstein condensates (BECs) by sweeping three laser beams through the BEC. If one of the beams is in the first order (TEM10) Hermite-Gaussian mode, its amplitude has a transversal $\pi$ phase slip which can be transferred to the atoms creating a soliton. Using this method it is possible to circumvent the restriction set by the diffraction limit inherent to conventional methods such as phase imprinting. The method allows one to create multicomponent (vector) solitons of the dark-bright form as well as the dark-dark combination. In addition it is possible to create in a controllable way two or more dark solitons with very small velocity and close to each other for studying their collisional properties.
\end{abstract}

Keywords: cold atoms, atomic Bose-Einstein condensates, solitons

PACS: 03.75.Hh

\section{Introduction}

Atomic Bose-Einstein condensates (BECs) have received a great deal of interest since they were first produced a decade ago [1-3]. They can exhibit various topological excitations, such as vortices and solitons. The dynamics of solitons in elongated BECs [4] is the atom-optics version of the nonlinear propagation of light pulses in optical fibres [5]. The BEC offers a remarkable freedom in terms of controlling the physical parameters such as dimensionality and even the sign of the strength of the atom-atom interaction [4].

Solitons in BECs can be of both dark and bright type. Dark solitons are formed in BECs with repulsive interaction between the atoms [4]. For completely dark solitons the condensate wavefunction is zero at the centre and changes its sign when crossing the central point, i. e. the condensate wave function has an infinitely steep $\pi$ phase slip at the centre [4]. On the other hand, bright solitons are formed in BECs with repulsive interaction between the atoms. The wave function of the BEC is then localised at the centre [4] and goes to zero further away from this point. Dark solitons which manifest

\footnotetext{
${ }^{*}$ The report presented at the 37th Lithuanian National Physics Conference, 11-13 June 2007, Vilnius, Lithuania.
}

themselves as a density minimum moving with a constant speed against a uniform background density, as well as bright solitons which are shape preserving wave packets, have both been experimentally realised [6-9]. The dynamics of solitons in BECs has been extensively studied. This has included investigations of the stability properties [10], as well as soliton dynamics in inhomogeneous clouds [11], in multicomponent BECs $[12,13]$, and in supersonic flow [14]. Solitons can be created in various ways with a variable degree of controllability, e. g., by colliding clouds of BEC [15-17] or engineering the density $[18,19]$.

Traditionally dark solitons in BECs are created using phase imprinting $[6,7,20-22]$, where a part of the condensate cloud is illuminated by a far detuned laser pulse in order to induce a sharp $\pi$ phase slip in the wave function. The subsequent dynamics can indeed develop solitons $[6,7]$. There are, however, some rather severe drawbacks with such a method of phase engineering. The resolution of the required phase slip is naturally restricted by the diffraction limit, i. e. the width of the phase slip should be larger than an optical wavelength. Furthermore, the phase imprinting does not produce a density minimum characteristic of the dark solitons in the region of the phase change. Hence completely dark 
(a)

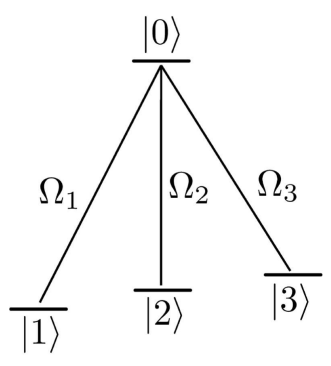

(b)

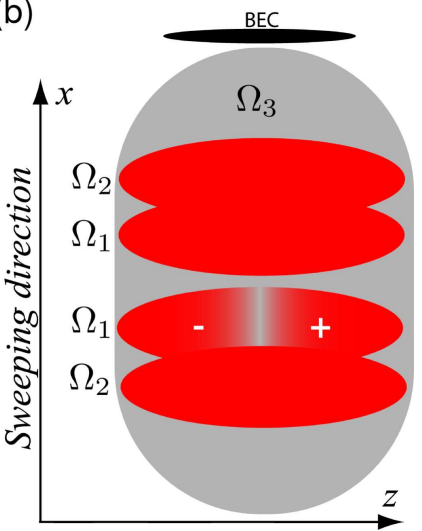

Fig. 1. (a) The level scheme for the three laser beams $\Omega_{i}(i=1,2$, 3). (b) The sequence of laser beams being swept through the BEC involves a preparation stage $\Omega_{2} \rightarrow \Omega_{1}$ and a final stage $\Omega_{1} \rightarrow \Omega_{2}$ which engineers the phase and density of the BEC to produce a soliton.

stationary solitons are difficult to achieve, which consequently results in so called grey moving solitons with a shallow density dip.

It is of a significant interest to be able to create slowly moving, or even completely stationary solitons in order to test for instance their scattering properties, where the shapes of the colliding solitons are preserved. In addition, a relative spatial shift is expected. This spatial shift, however, can only be detected for extremely slow solitons due to the inherent logarithmic dependence of the spatial shift on the relative velocity between the solitons $[23,24]$. The standard phase imprinting also inevitably creates phonons in the trapped cloud because the constructed initial state is not the exact soliton solution largely due to the missing density notch $[6,7]$.

In this paper we show how states which have the required phase slip and density profile for solitons can be created by sweeping three laser beams through an elongated BEC as shown in Fig. 1. If one of the beams is in the first order (TEM10) Hermite-Gaussian mode, its amplitude has a transversal $\pi$ phase slip which will be transferred to atoms thus producing a soliton. More importantly, with a sequence of three laser beams it is possible to circumvent the restriction set by the diffraction limit. The laser fields reshape an atomic wave function so that it acquires a zero-point. This leads to a hole in the atomic density, the width of which is only limited by the intensity ratios between the incident laser beams due to the geometric nature [25] of the process. The formation of the hole is accompanied by a step-like (infinitely sharp) $\pi$ phase slip in the atomic wave function when crossing the zero-point. The method is particularly useful for creating multicomponent (vector) soli-

tons of the dark-bright form as well as the dark-dark combination. In addition it is possible to create in a controllable way two or more slowly moving dark solitons close to each other for studying their collisional properties.

\section{Formulation}

\subsection{Outline of the proposed setup}

Consider a cigar-shape atomic BEC elongated in the $z$ direction. To create solitons in the BEC, we propose to sweep three incident laser beams across the condensate. The laser beams interact with the condensate atoms in a tripod configuration $[25,26]$, i. e. the atoms are characterized by three ground states $|1\rangle,|2\rangle,|3\rangle$ and an excited state $|0\rangle$. The $j$ th laser drives resonantly the atomic transition between the ground state $|j\rangle$ and the excited state $|0\rangle$, see Fig. 1(a). Initially the atoms forming the BEC are prepared in the hyperfine ground state $|1\rangle$. Subsequently the lasers are swept through the BEC in the $x$ direction, i. e. perpendicular to the longitudinal axis $z$ of the condensate.

The sweeping process is made of two stages depicted in Fig. 1(b). In the first stage the lasers 1 and 2 are applied in a counter-intuitive sequence to transfer adiabatically the atoms from the ground state $|1\rangle$ to another ground state $|2\rangle$. If an additional laser 3 is on during the first stage, a partial transfer of atoms from the ground states $|1\rangle$ to $|2\rangle$ is possible [26]. In that case a coherent superposition of states $|1\rangle$ and $|2\rangle$ is created after completing the first stage. In the second stage, the lasers 1,2, and 3 are applied once again to transfer atoms from the state $|2\rangle$ back to the state $|1\rangle$ and from the state $|1\rangle$ to the state $|2\rangle$. If the amplitude of one of these lasers $\Omega_{1}$ or $\Omega_{2}$ changes the sign at $z=z_{0}$, the BEC picks up a $\pi$ phase shift at this point after the sweeping, and a soliton can be formed. This is the case e.g. if one of the beams is the first order Hermite-Gaussian beam centred at $z=0$.

It is important to realize that at least two laser fields are needed to complete the adiabatic transfer of population between the ground states. Therefore the adiabaticity can be violated in the vicinity of the point $z=z_{0}$ where one of the Rabi frequencies $\Omega_{1}$ or $\Omega_{2}$ goes to zero. Inclusion of the third (support) laser 3 helps to avoid such a violation of the adiabaticity. In fact the atoms would experience absorption in the vicinity of $z=z_{0}$ if the support laser 3 was missing during the second stage. 
It should be mentioned that there are similar previous proposals for creating vortices in a BEC via the two-laser Raman processes involving the transfer of an optical vortex to the atoms [27-29]. In these schemes the lasers are far detuned from the single-photon resonance to avoid the absorption at the vortex core. In our scheme the lasers are in an exact single-photon resonance, so the use of the third (support) laser is essential to avoid the losses. An advantage of the resonant scheme is that an efficient and complete population transfer is possible between the hyperfine ground states, whereas in the non-resonant case only a fraction of population can be transferred [29].

\subsection{Hamiltonian for a tripod atom}

Let us now provide a quantitative description of our scheme. The $j$ th laser beam is characterized by the complex Rabi frequency $\tilde{\Omega}_{j}=\Omega_{j} \exp \left(\mathrm{ik}_{j} \cdot \mathbf{r}+\mathrm{i} S_{j}\right)$, with $j=1,2,3$, where $\Omega_{j}$ is the real amplitude, the phase being comprised of the local phase $\mathbf{k}_{j} \cdot \mathbf{r}$ as well as the global (distance-independent) phase $S_{j}$. In what follows, the Rabi frequencies $\Omega_{2}$ and $\Omega_{3}$ are considered to be positive: $\Omega_{2}>0, \Omega_{3}>0$. Yet, the Rabi frequency $\Omega_{1}$ is allowed to be negative. This makes it possible to include an additional $\pi$ phase shift in the spatial profile of the first beam when crossing the zeropoint at $z=z_{0}$.

The electronic Hamiltonian of a tripod atom reads in the interaction representation:

$$
\hat{H}_{\mathrm{e}}=-\hbar\left(\tilde{\Omega}_{1}|0\rangle\left\langle 1\left|+\tilde{\Omega}_{2}\right| 0\right\rangle\left\langle 2\left|+\tilde{\Omega}_{3}\right| 0\right\rangle\langle 3|\right)+\text { H. c. }
$$

The tripod atoms have two degenerate dark states $\left|D_{1}\right\rangle$ and $\left|D_{2}\right\rangle$ of zero eigenenergy $\left(\hat{H}_{\mathrm{e}}\left|D_{n}\right\rangle=0\right)$ containing no excited-state contribution $[25,26]$,

$$
\begin{array}{r}
\left|D_{1}\right\rangle=\frac{1}{\sqrt{1+\zeta^{2}}}\left(|1\rangle^{\prime}-\zeta|2\rangle^{\prime}\right), \\
\left|D_{2}\right\rangle=\frac{1}{\sqrt{1+\zeta^{2}}}\left[\xi_{3}\left(\zeta|1\rangle^{\prime}+|2\rangle^{\prime}\right)\right. \\
\left.-\xi_{2}\left(1+\zeta^{2}\right)|3\rangle^{\prime}\right],
\end{array}
$$

where $|j\rangle^{\prime}=|j\rangle \exp \left[\mathrm{i}\left(\mathbf{k}_{3}-\mathbf{k}_{j}\right) \cdot \mathbf{r}+\mathrm{i}\left(S_{3}-S_{j}\right)\right]$ (with $j=1,2,3)$ are the modified atomic state vectors accommodating the phases of the incident laser fields, $\zeta=\Omega_{1} / \Omega_{2}$ is the ratio between the Rabi frequencies of the first and second fields, and $\xi_{j}$ are the normalised Rabi frequencies $(j=1,2,3)$,

$$
\xi_{j}=\frac{\Omega_{j}}{\Omega}, \quad \Omega=\sqrt{\Omega_{1}^{2}+\Omega_{2}^{2}+\Omega_{3}^{2}},
$$

with $\xi_{3}>0$ and $-\infty<\zeta<+\infty$. The atomic dark states $\left|D_{1}\right\rangle$ and $\left|D_{2}\right\rangle$ depend on the centre of mass coordinate $\mathbf{r}$ through the spatial dependence of the Rabi frequencies $\Omega_{j}$ and state vectors $|j\rangle^{\prime}$.

\subsection{General equations of motion}

The full atomic state vector of a multicomponent BEC is $|\Phi(\mathbf{r}, t)\rangle=\sum_{j=1}^{4}|j\rangle \Psi_{j}(\mathbf{r}, t)$, where the constituent wave functions $\Psi_{j}(\mathbf{r}, t)$ describe the translational motion of the BEC in the internal state $|j\rangle$ of the tripod scheme. The wave functions $\Psi_{j}(\mathbf{r}, t)$ obey a multicomponent Gross-Pitaevski equation of the form

$$
\mathrm{i} \hbar \frac{\partial}{\partial t}|\Phi(\mathbf{r}, t)\rangle=\left[\frac{1}{2 M} \nabla^{2}+\hat{H}_{\mathrm{e}}+\hat{V}\right]|\Phi(\mathbf{r}, t)\rangle,
$$

where $\hat{H}_{\mathrm{e}}$ from Eq. (1) describes the light-induced transitions between the different internal states of atoms. The diagonal operator

$$
\hat{V}=\sum_{l>j=0}^{3}\left(V_{j}+g_{j l}\left|\Psi_{l}\right|^{2}\right)|j\rangle\langle j|
$$

accommodates the trapping potential $V_{j}(\mathbf{r})$ for the $j$ th internal state, as well as the nonlinear interaction between the components $j$ and $l$ characterized by the strength $g_{j l}=4 \pi \hbar^{2} a_{j l} / m$, with $a_{j l}$ being the corresponding scattering length.

\section{Time-evolution of the atom-light system}

\subsection{Adiabatic approximation for the dark states}

We shall apply the adiabatic approximation $[25,30$, 31] under which atoms evolve within their dark-state manifold during the sweeping. This is legitimate if the total Rabi frequency $\Omega$ is sufficiently large compared to the inverse sweeping duration $\tau_{\text {sweep }}^{-1}$. The full atomic state vector can then be expanded as $|\Phi(\mathbf{r}, t)\rangle=$ $\sum_{n=1}^{2} \Psi_{n}^{(D)}(\mathbf{r}, t)\left|D_{n}(\mathbf{r}, t)\right\rangle$, where a composite wavefunction $\Psi_{n}^{(D)}(\mathbf{r})$ describes the translational motion of an atom in the dark state $\left|D_{n}(\mathbf{r}, t)\right\rangle$. The atomic centre of mass motion is thus represented by a two-component wave function

$$
\Psi=\left(\begin{array}{c}
\Psi_{1}^{(D)} \\
\Psi_{2}^{(D)}
\end{array}\right)
$$

obeying the following equation of motion [25]:

$$
\mathrm{i} \hbar \frac{\partial}{\partial t} \Psi=\left[\frac{1}{2 M}(-\mathrm{i} \hbar \nabla-\mathbf{A})^{2}+V(\mathbf{r})+\phi-\beta\right] \Psi,
$$


where the effective vector potential $\mathbf{A}$ and the matrix $\beta$ are the $2 \times 2$ matrices appearing due to the spatial and temporal dependence of the dark states, $\mathbf{A}_{n, m}=$ $\mathrm{i} \hbar\left\langle D_{n}(\mathbf{r}, t)|\nabla| D_{m}(\mathbf{r}, t)\right\rangle$ and $\beta_{n, m}=\mathrm{i} \hbar\left\langle D_{n}(\mathbf{r}, t)\right| \times$ $\times \partial / \partial t\left|D_{m}(\mathbf{r}, t)\right\rangle$. The former $\mathbf{A}$ is known as the Mead-Berry connection [32,33], whereas the latter matrix $\beta$ is responsible for the geometric phase [34]. The $2 \times 2$ matrix $\phi$ is the effective trapping potential (explicitly presented in Ref. [25]) appearing due to the spatial dependence of the dark states. Assuming that all three beams co-propagate $\left(\mathbf{k}_{1} \approx \mathbf{k}_{2} \approx \mathbf{k}_{3}\right)$, the effective vector potential [25] reduces to

$$
\mathbf{A}=\hbar \frac{\xi_{3}}{1+\zeta^{2}} \nabla \zeta\left(\begin{array}{rr}
0 & \mathrm{i} \\
-\mathrm{i} & 0
\end{array}\right) .
$$

Lastly, the $2 \times 2$ matrix $V$ originating from the operator $\hat{V}$, Eq. (6), accommodates the trapping potential for the dark states [25] as well as the atom-atom coupling.

\subsection{Time-evolution during the sweeping}

Suppose the incident laser beams are swept through a trapped BEC along the $x$ axis with a velocity $\mathbf{v}$, as shown in Fig. 1(b). This can be done either by shifting in the transversal $(x)$ direction the laser beams propagating along the $y$ axis or by applying a set of laser pulses of the appropriate shape and sequence propagating in the $x$ direction. In the latter case, the sweeping velocity $v$ will coincide with the speed of light. In both cases the adiabatic dark states depend on time in the following way: $\left|D_{n}(\mathbf{r}, t)\right\rangle \equiv\left|D_{n}\left(\mathbf{r}^{\prime}\right)\right\rangle$, where $\mathbf{r}^{\prime}=\left(x^{\prime}, y, z\right) \equiv(x-v t, y, z)$ is the atomic coordinate in the frame of the moving laser fields. Let us assume that the time $\tau_{\text {sweep }}=d / v$ it takes to sweep the laser beams through a BEC of the width $d$, is small compared to the time associated with the BEC chemical potential $\tau_{\mu}=\hbar / \mu$ which is typically of the order of $10^{-5} \mathrm{~s}$. In that case one can neglect the dynamics of the atomic centre of mass during the sweeping. Consequently the time evolution of the multicomponent wave function during the sweeping is governed by the matrix-term $\beta=-v A_{x}$ featured in Eq. (8), giving

$$
\mathrm{i} \hbar \partial_{t} \Psi=v A_{x} \Psi
$$

where $A_{x}$ is the effective vector potential along the sweeping direction.

In passing we note that the subsequent time evolution of the BEC after the two-stage sweeping will be described by the general Gross-Pitaevski equation (5) with the light fields off $\left(\hat{H}_{\mathrm{e}}=0\right)$, as we shall do in Section 4.
Returning to Eq. (10), since $v A_{x}$ commutes with itself at different times, one can relate the wave function $\Psi(t)$ at a final time $t=t_{f}$ to the one at the initial time $t=t_{i}$ as

$$
\Psi\left(\mathbf{r}, t_{f}\right)=\exp (-\mathrm{i} \Theta) \Psi\left(\mathbf{r}, t_{i}\right),
$$

where the exponent $\Theta$ is a $2 \times 2$ Hermitian matrix

$$
\Theta=\frac{1}{\hbar} \int_{t_{i}}^{t_{f}} A_{x}(\mathbf{r}-\mathbf{v} t) v \mathrm{~d} t=\frac{1}{\hbar} \int_{x_{f}}^{x_{i}} A_{x}\left(\mathbf{r}^{\prime}\right) \mathrm{d} x^{\prime}
$$

and the integration is over the sweeping path $\mathbf{r}^{\prime}=$ $(x-v t, y, z)$ from $x_{f}=x-v t_{f}$ to $x_{i}=x-v t_{i}$. In most cases of interest the initial and final times can be considered to be sufficiently remote, so that the spatial integration can be from $x_{f}=-\infty$ to $x_{i}=+\infty$.

\subsubsection{The first stage}

Let us now analyse the proposed two-stage setup in more details. In the first stage both Rabi frequencies $\Omega_{1}$ and $\Omega_{2}$ are positive. The lasers 1 and 2 are applied in a counterintuitive order (see Fig. 1(b)), where the ratio $\zeta=\Omega_{1} / \Omega_{2}$ changes from $\zeta\left(t_{i}^{\prime}\right)=0$ to $\zeta\left(t_{f}^{\prime}\right)=+\infty$. On the other hand, the laser 3 is dominant for both the initial and final times where $\xi_{3}=\Omega_{3} / \Omega=1$. Initially the BEC has the wave function $\Psi(\mathbf{r})$ and is in the internal atomic ground state $|1\rangle$ which coincides with the first dark state at the initial time $t_{i}^{\prime}$, i.e. $\left|D_{1}\left(\mathbf{r}, t_{i}^{\prime}\right)\right\rangle=|1\rangle$. The full initial atomic state vector is therefore $\left|\Phi\left(\mathbf{r}, t_{i}^{\prime}\right)\right\rangle=\Psi(\mathbf{r})\left|D_{1}\left(\mathbf{r}, t_{i}^{\prime}\right)\right\rangle$. This provides the following initial condition for the multicomponent wave function:

$$
\Psi\left(\mathbf{r}, t_{i}^{\prime}\right)=\left(\begin{array}{c}
\Psi(\mathbf{r}) \\
0
\end{array}\right) .
$$

Equations (9) and (11)-(13) yield the multicomponent wave function after the first stage

$$
\Psi\left(\mathbf{r}, t_{f}^{\prime}\right)=\Psi(\mathbf{r})\left(\begin{array}{r}
\cos \beta \\
-\sin \beta
\end{array}\right),
$$

where

$$
\beta=\int_{-\infty}^{+\infty} \xi_{3} \frac{\partial \arctan \zeta}{\partial x^{\prime}} \mathrm{d} x^{\prime}
$$

is the mixing angle between the dark states acquired in the first stage.

Suppose we have the following laser beams. The second beam $\Omega_{2}$ is a Gaussian beam characterized by a waist $\sigma_{z}$ in the $z$ direction. The beam is centred at 


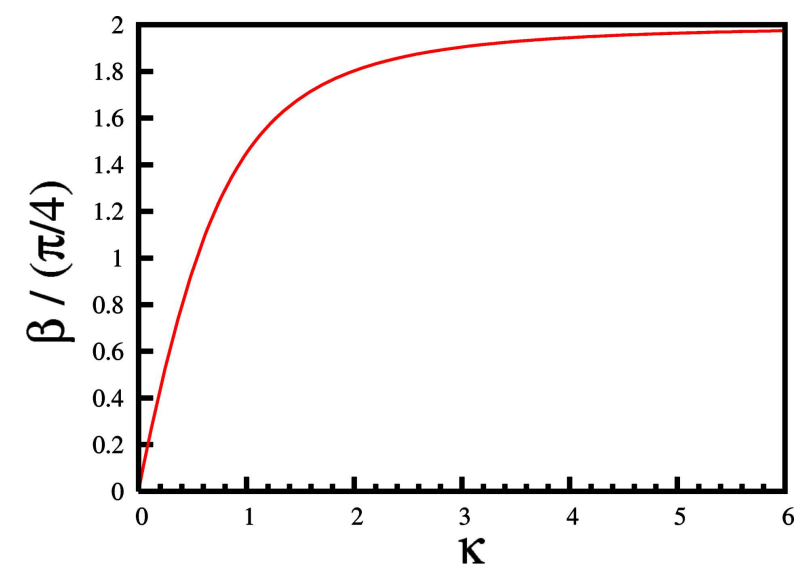

Fig. 2. Dependence of the mixing angle $\beta$ on the relative amplitude of the third beam $\kappa$. The spatial separation between the first and the second beams is taken to be $2 \Delta=1.2 \sigma_{x}$ where $\sigma_{x}$ is the width of the beams in the sweeping direction.

$x^{\prime}=\bar{x}+\Delta$ in the sweeping direction and at $z=0$ in the $z$ direction,

$$
\Omega_{2}=A \mathrm{e}^{-z^{2} / \sigma_{z}^{2}-\left(x^{\prime}-\bar{x}-\Delta\right)^{2} / \sigma_{x}^{2}} .
$$

The first beam $\Omega_{1}$ is characterized by the same amplitude $A$, the same waist $\sigma_{z}$, and the same width $\sigma_{x}$. Yet it is centred at $x^{\prime}=\bar{x}-\Delta$ in the sweeping direction, where $2 \Delta$ is the separation between the two beams. The beam waists should be of the order of the condensate length (or larger) in the $z$ direction, so that the whole condensate is illuminated by the beams.

The third beam is considered to change little along the sweeping direction $x$. Furthermore, it has the same width $\sigma_{z}$ in the $z$ direction as the first two beams,

$$
\Omega_{3}=A \kappa \mathrm{e}^{-z^{2} / \sigma_{z}^{2}} .
$$

The first stage is aimed at creating a superposition of states $|1\rangle$ and $|2\rangle$. Since we take all the beams to be Gaussian beams characterized by the same widths $\sigma_{z}$, the Rabi frequency ratios $\Omega_{2} / \Omega_{1}$ and $\Omega_{3} / \Omega_{1}$ have no $z$ dependence. As a result the acquired mixing angle $\beta$ has no $z$ dependence, i.e. it is uniform along the BEC. The magnitude of $\beta$ depends on the relative intensity of the third laser. If the third laser is weak $\left(\xi_{3}=\Omega_{3} / \Omega \rightarrow 0\right.$ at the crossing point where $\zeta=\Omega_{1} / \Omega_{2}=1$ ), the mixing between the states $|1\rangle$ and $|2\rangle$ is small: $\beta \ll 1$. On the other hand, if the Rabi frequency $\Omega_{3}$ is comparable with $\Omega_{1}$ and $\Omega_{2}$ at the crossing point where $\zeta=\Omega_{1} / \Omega_{2}=1$, the mixing can be close to its maximum: $\beta \approx \pi / 4$. In this way, one can control the mixing angle by changing the intensity of the third beam, as one can see from Fig. 2 .

\subsubsection{The second stage}

In the second stage the Rabi frequency $\Omega_{1}$ can be both positive and negative depending on the transversal coordinate $z$. The laser 1 is now applied first, so that the ratio $\zeta=\Omega_{1} / \Omega_{2}$ changes from $\zeta\left(t_{i}\right)= \pm \infty$ to $\zeta\left(t_{f}\right)=$ 0 in the second stage. Again the third laser dominates for the initial and final times: $\Omega_{3} / \Omega=1$. The second stage takes place immediately after completing the first stage, so the multicomponent wave function of the first stage (14) serves as an initial condition for the second stage.

Equations (11), (12), and (14) together with (2) and (3) yield the total state vector after the second stage:

$$
\begin{aligned}
& \left|\Phi\left(\mathbf{r}, t_{f}\right)\right\rangle= \\
& |1\rangle \Psi(\mathbf{r})\left(\sin \gamma \cos \beta-\mathrm{e}^{\mathrm{i} \nu_{12}} \cos \gamma \sin \beta\right) \\
& -|2\rangle \Psi(\mathbf{r})\left(\cos \gamma \cos \beta+\mathrm{e}^{\mathrm{i} \nu_{12}} \sin \gamma \sin \beta\right),
\end{aligned}
$$

where $\nu_{12}=S_{1}-S_{2}+S_{2}^{\prime}-S_{1}^{\prime}$ is the phase mismatch between the Rabi frequencies $\Omega_{1}$ and $\Omega_{2}$ in the first and second stages. The resulting mixing angle acquired in the second stage is

$$
\gamma \equiv \gamma_{z}=\int_{-\infty}^{+\infty}\left(1-\xi_{3}\right) \frac{\partial \arctan \zeta}{\partial x^{\prime}} \mathrm{d} x^{\prime} .
$$

If the first and second lasers are weak $\left(\Omega_{3} / \Omega \rightarrow 1\right.$ at the crossing point where $\zeta=\Omega_{1} / \Omega_{2}=1$ ), the mixing angle is small: $\gamma_{z} \ll 1$. On the other hand, if first and second lasers are strong at this point, we have $\gamma_{z} \rightarrow$ $\mp \pi / 2$. The change in sign of $\gamma_{z}$ will introduce a phase shift which is needed to create solitons.

In the second stage the first beam $\Omega_{1}$ is a first-order (in the $z$ direction) Hermite-Gaussian beam centred at $z=0$ and $x^{\prime}=\tilde{x}+\tilde{\Delta}$ :

$$
\Omega_{1}=A \frac{z}{B} \mathrm{e}^{-z^{2} / \sigma_{z}^{2}-\left(x^{\prime}-\tilde{x}-\tilde{\Delta}\right)^{2} / \sigma_{x}^{2}},
$$

where $z= \pm B$ represents a distance where $\Omega_{1}= \pm \Omega_{2}$ for $x^{\prime}=\tilde{x}$. In most cases of interest the distance $B$ is much smaller than the waist of the beams: $B \ll \sigma_{z}$. The second beam $\Omega_{2}$ is the ordinary Gaussian beam centred at $z=0$ along the BEC and $x^{\prime}=\tilde{x}-\tilde{\Delta}$ in the sweeping direction:

$$
\Omega_{2}=A \mathrm{e}^{-z^{2} / \sigma_{z}^{2}-\left(x^{\prime}-\tilde{x}+\tilde{\Delta}\right)^{2} / \sigma_{x}^{2}},
$$

where $2 \tilde{\Delta}$ is the separation between the two beams. The ratio between the Rabi frequencies reads then:

$$
\zeta=\frac{\Omega_{1}}{\Omega_{2}}=\frac{z}{B} \mathrm{e}^{4 \tilde{\Delta}\left(x^{\prime}-\tilde{x}\right) / \sigma_{x}^{2}} .
$$


Equation (22) provides the following limiting cases:

$$
\zeta \equiv \zeta\left(z, x^{\prime}\right)=\left\{\begin{array}{ccc}
0, & \text { for } & x^{\prime} \rightarrow+\infty \\
\pm \infty, & \text { for } & x^{\prime} \rightarrow-\infty
\end{array}\right.
$$

Finally let us determine the crossing point where $\Omega_{1}=$ $\Omega_{2}$. Using Eq. (22), the condition $|\zeta|=1$ yields the crossing point $x^{\prime}=x_{\mathrm{cr}}^{\prime}$ for a fixed $z$ coordinate:

$$
x_{\mathrm{cr}}^{\prime}=\tilde{x}+\frac{\sigma^{2}}{4 \tilde{\Delta}} \ln \frac{z}{B} \text {. }
$$

Specifically, if $z=B$, the crossing point is $x_{\mathrm{cr}}^{\prime}=\tilde{x}$. Since $B \ll \sigma$, the Rabi frequencies at $z=B$ and $x^{\prime}=\tilde{x}$ are

$$
\Omega_{1}=\Omega_{2} \approx A \mathrm{e}^{-\tilde{\Delta}^{2} / \sigma_{x}^{2}} .
$$

In the next subsection we shall analyse in more detail the multicomponent wave function after completing the second stage.

\subsection{Multicomponent wave function alter the sweeping}

Suppose that there is no phase mismatch between the lasers of the first and second stages: $\nu_{12}=0$. In that case Eq. (18) yields

$$
\left|\Phi\left(\mathbf{r}, t_{f}\right)\right\rangle=\Psi(\mathbf{r})\left[-\sin \left(\gamma_{z}-\beta\right)|1\rangle+\cos \left(\gamma_{z}-\beta\right)|2\rangle\right] .
$$

If $\beta=0$, the second component is populated after the first stage. After the whole sweeping the state vector then takes the form

$$
\left|\Phi\left(\mathbf{r}, t_{f}\right)\right\rangle=\Psi(\mathbf{r})\left[-\sin \gamma_{z}|1\rangle+\cos \gamma_{z}|2\rangle\right] .
$$

In this case the first component alters the sign at $z=z_{0}$ where the Rabi frequency $\Omega_{1}$ or $\Omega_{2}$ (and hence $\gamma_{z}$ ) crosses the zero-point. On the other hand, the second component is maximum at this point and symmetrically decays to zero away from this point. Such a multicomponent wave function has a shape close to that of a soliton of the dark-bright form (see Fig. 3). This will indeed lead to the formation of such a soliton, as we shall see from the analysis of the subsequent time-evolution presented in the next Section.

On the other hand, $\beta=\pi / 4$ corresponds to the case where both components are initially populated with equal probabilities. Thus we have after the sweeping:

$$
\begin{aligned}
\left|\Phi\left(\mathbf{r}, t_{f}\right)\right\rangle=-\Psi(\mathbf{r})[ & -\sin \left(\gamma_{z}-\pi / 4\right)|1\rangle \\
+ & \left.\sin \left(\gamma_{z}+\pi / 4\right)|2\rangle\right] .
\end{aligned}
$$

In that case both components of the wave function acquire a $\pi$ phase shift in a vicinity of $z=z_{0}$ where

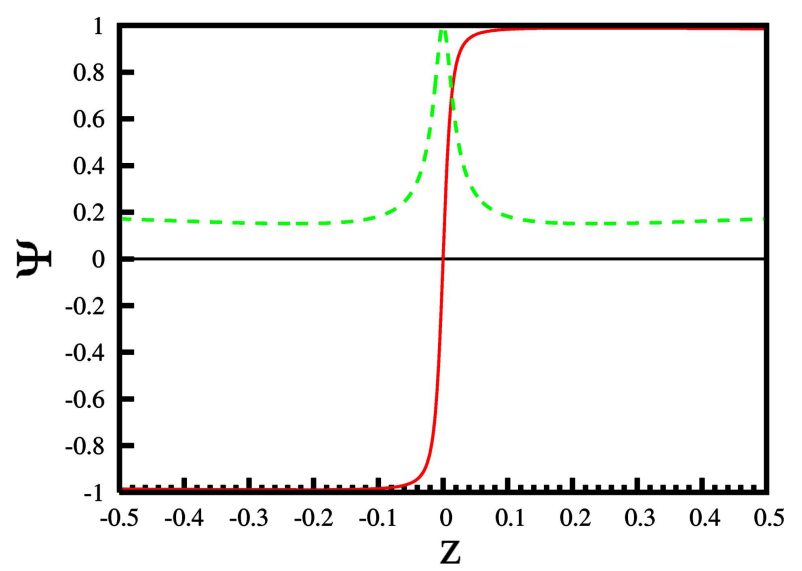

Fig. 3. Multicomponent wave function after completing the second stage in the case where the second component is populated after the first stage $(\beta=0)$ and there is no phase mismatch between the lasers of the first and second stages $\left(\nu_{12}=0\right)$. The second and third laser beams are taken to be the Gaussian beams with equal widths $\sigma_{z}$. The first laser beam is the first order Hermite-Gaussian beam with same width $\sigma_{z}$. The parameters used are $2 \tilde{\Delta} / \sigma_{x}=1.2$, $B / \sigma_{z}=0.1$, and $\kappa=0.1$. The wave function of the first (second) component is plotted in a solid (dashed) line.

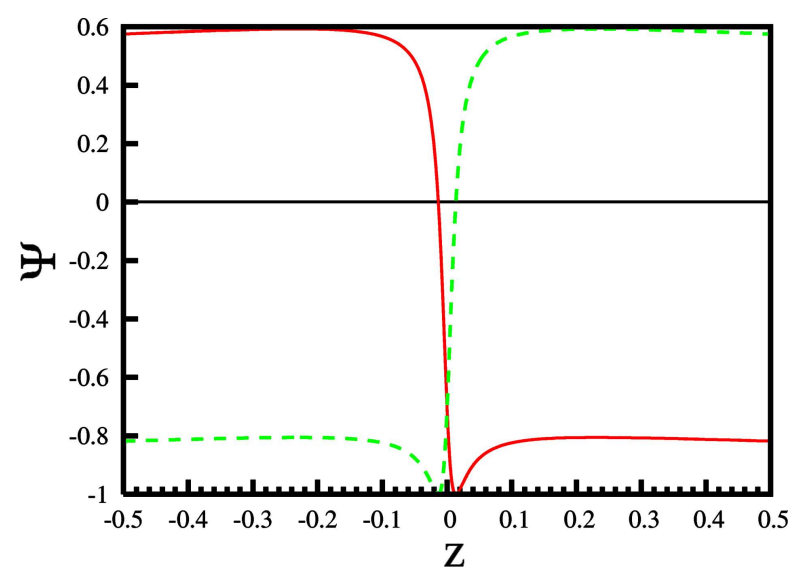

Fig. 4. Multicomponent wave function after completing the second stage in the case where both components are initially populated after the first stage ( $\beta=\pi / 4)$ and there is no phase mismatch between the lasers of the first and second stages $\left(\nu_{12}=0\right)$. The second and third laser beams are taken to be the Gaussian beams with equal widths $\sigma_{z}$. The first laser beam is the first order HermiteGaussian beam with same width $\sigma_{z}$. The parameters used are $2 \tilde{\Delta} / \sigma_{x}=1.2, B / \sigma_{z}=0.1$, and $\kappa=0.1$. The wave function of the first (second) component is plotted in a solid (dashed) line.

$\Omega_{1}=0$, as one can see clearly in the Fig. 4 . Note that the zero-points of each component are slightly shifted with respect to each other. This makes it possible to produce two-component dark-dark solitons oscillating around each other, as we shall see in the following Section. 
If $\beta=\pi / 4$, yet there is a $\pi / 2$ phase mismatch $\left(\nu_{12}=\pi / 2\right)$, Eq. (18) reduces to

$$
\left|\Phi\left(\mathbf{r}, t_{f}\right)\right\rangle=-\Psi(\mathbf{r}) \mathrm{e}^{\mathrm{i} \gamma_{z}} \frac{1}{\sqrt{2}}[|2\rangle-\mathrm{i}|1\rangle] .
$$

In that case both components are characterized by the same spatial modulation $\exp \left(\mathrm{i} \gamma_{z}\right)$ and have a relative phase $\pi / 2$ after the sweeping. Therefore both components initially have the same velocity distribution proportional to $\nabla \gamma_{z}$. Furthermore, there is no hole in the atomic density of neither component after the sweeping, like is the case in the phase imprinting techniques.

In this way, the creation of solitons can be controlled by changing the mixing angle $\beta$ and the phase mismatch $\nu_{12}$

\section{Subsequent dynamics and soliton formation}

The optical preparation of the initial state of the twocomponent Bose-Einstein condensate described in the previous section, is fast compared to any characteristic dynamics in the Bose-Einstein condensate. This is the case if the time $\tau_{\text {sweep }}=d / v$ it takes to sweep the laser beams through a BEC of the width $d$ is small compared to the time associated with the BEC chemical potential $\tau_{\mu}=\hbar / \mu$ which is typically of the order of $10^{-5} \mathrm{~s}$. With the prepared initial state and for sufficiently low temperatures we can therefore describe the subsequent dynamics using a two-component GrossPitaevskii equation [12]

$$
\mathrm{i} \hbar \frac{\partial}{\partial t} \Psi_{1}=
$$

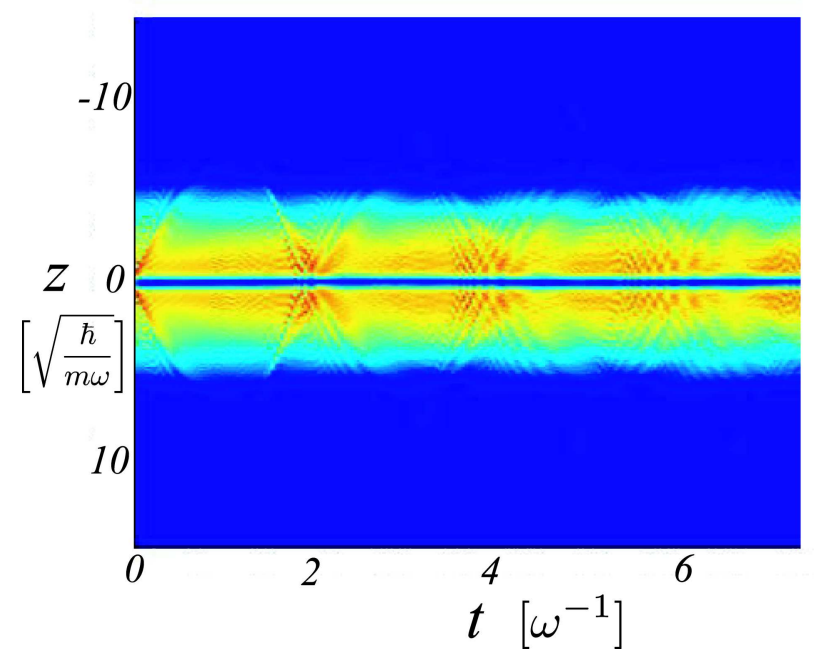

(a)

$$
\begin{aligned}
& =\left[-\frac{\hbar^{2}}{2 m} \nabla^{2}+V(z)+g_{11}\left|\Psi_{1}\right|^{2}+g_{12}\left|\Psi_{2}\right|^{2}\right] \Psi_{1}, \\
& \mathrm{i} \hbar \frac{\partial}{\partial t} \Psi_{2}= \\
& =\left[-\frac{\hbar^{2}}{2 m} \nabla^{2}+V(z)+g_{22}\left|\Psi_{2}\right|^{2}+g_{12}\left|\Psi_{1}\right|^{2}\right] \Psi_{2} .
\end{aligned}
$$

The external potential is here chosen to be quadratic in the $z$ direction,

$$
V(z)=\frac{1}{2} m \omega^{2} z^{2},
$$

where $\omega$ is the trap frequency and $m$ the atomic mass. The two-body interactions are described by

$$
g_{i j}=\frac{4 \pi \hbar^{2} a_{i j}}{m S}, \quad i, j=\{1,2\},
$$

with the scattering lengths $a_{i j}$ which represent the intra- and inter-collisional interactions between the atoms in the states 1 and 2. In Eq. (33) we have introduced the effective cross-section $S$ of the elongated cloud. Strictly speaking the elongated BoseEinstein condensate is three-dimensional. If, however, the transversal trapping is sufficiently strong, the dynamics can be considered effectively one-dimensional, as in Eqs. (31) and (32). This requires that the corresponding transversal ground state energy is much larger than the chemical potential of the condensate. We choose the normalisation as $\int \mathrm{d} z\left|\Psi_{i}(z)\right|^{2}=N_{i}$, where $N_{i}$ is the particle number in condensate $i(i=1,2)$.

With the initial states from the previous section we can simulate the dynamics of the Bose-Einstein

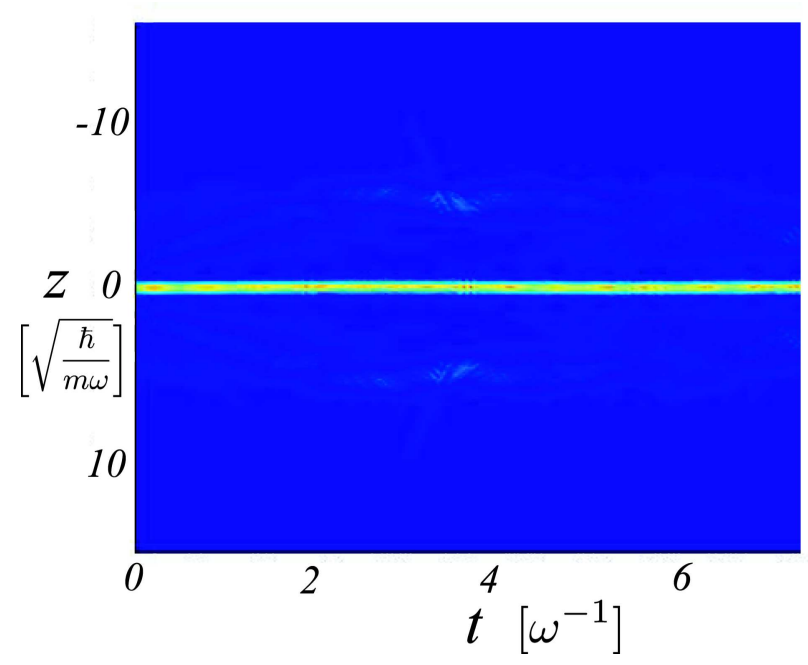

(b)

Fig. 5. The dark-bright soliton. The two figures show the one-dimensional density as a function of time for component 1 and 2 . The lighter (darker) colours correspond to higher (lower) atomic densities. 


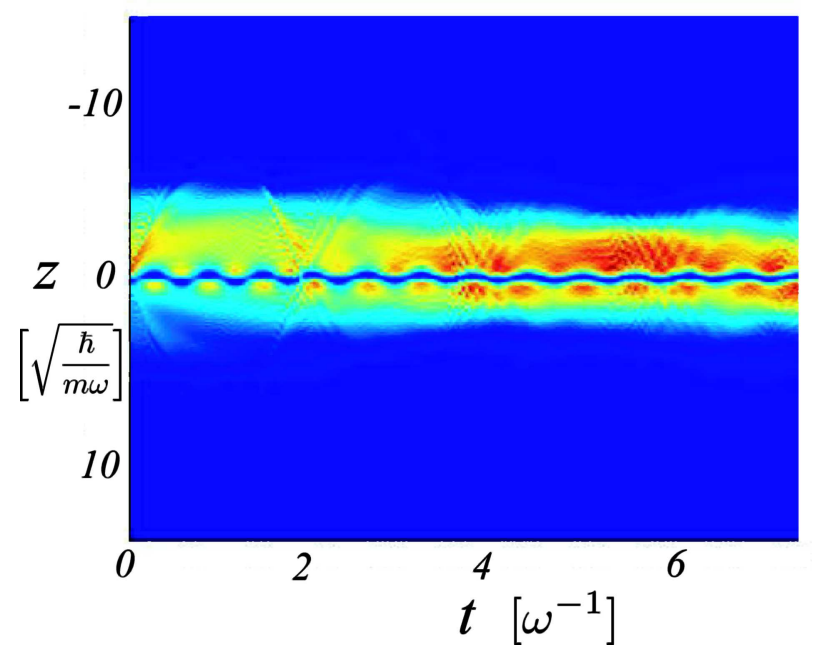

(a)

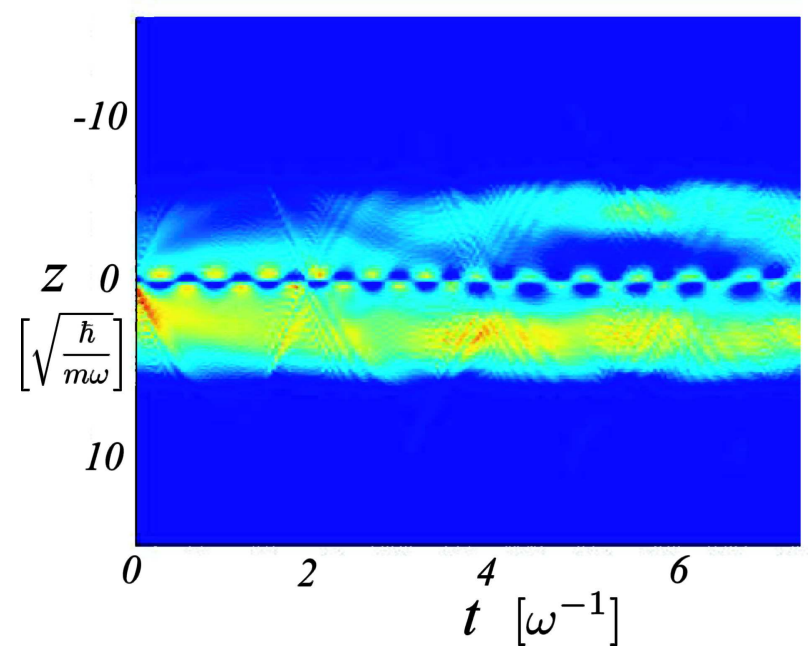

(b)

Fig. 6. The bound state dark-dark soliton. For sufficiently low initial soliton velocities the two dark solitons perform an oscillatory motion around each other. The figures show the one-dimensional atomic density as a function of time for component 1 and 2. The lighter (darker) colours correspond to higher (lower) densities.

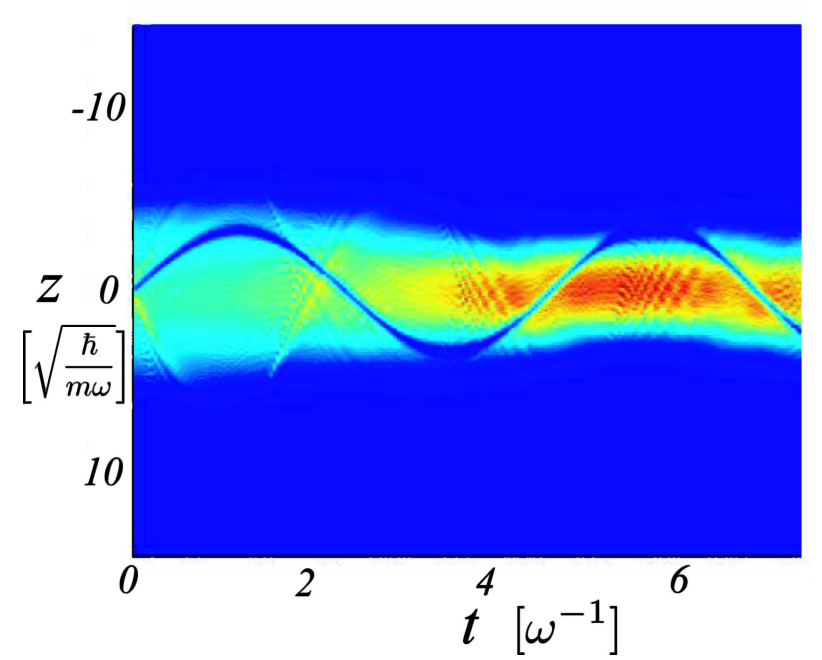

(a)

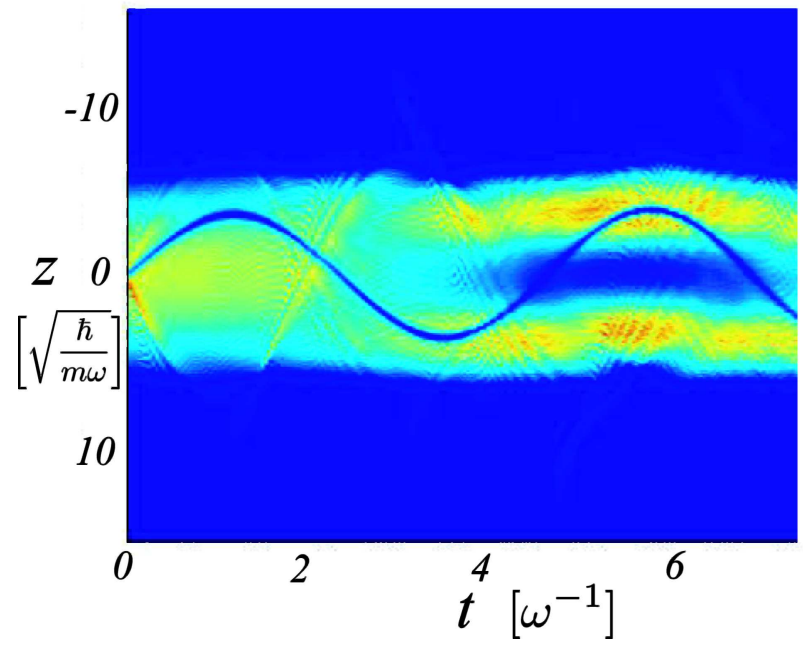

(b)

Fig. 7. The co-propagating dark-dark solitons. If the initial phase gradients of the two soliton solutions are chosen to be the same, the dark solitons propagate in unison. The two figures show the one-dimensional density as a function of time for component 1 and 2 . The lighter (darker) colours correspond to higher (lower) atomic densities.

condensate. We consider a condensate with $g_{11}: g_{12}$ : $g_{22}=1.0: 0.97: 1.03$, where $g_{12} N_{1}=286$ and $N_{1}=$ $N_{2}$. The unit of length is $\sqrt{\hbar /(m \omega)}$ and time is in units of $\omega^{-1}$. In Fig. 5 we show the dark-bright soliton dynamics whose initial state is prepared by choosing $\beta=$ 0 and $\nu_{12}=0$. The two-component system which has one dark soliton in component 1 and a bright soliton in component 2 is stable, i.e. the solitons are stationary. This shows that the initial state is indeed close to the exact soliton solution. If the initial state is prepared with $\beta=\pi / 4$ and $\nu_{12}=0$, on the other hand, the dynamics is strikingly different, see Fig. 6. In this case we create two dark solitons with opposite phase gradi- ents, hence there is an oscillatory motion, sometimes referred to as a soliton molecule. Such a bound state is only stable if the soliton velocities are low [12] which is indeed the case here. Alternatively, with $\beta=\pi / 4$ and $\nu_{12}=\pi / 2$, the solitons move in unison as shown in Fig. 7. The large oscillatory motion appearing in Fig. 7 stems from the fact that the condensate density is not homogeneous, hence the solitons experience an effective trap [11]. 


\section{Conclusions}

In summary, we have proposed a new method of creating solitons in elongated Bose-Einstein condensates (BECs) by sweeping three laser beams through the BEC. If one of the beams is the first order (TEM10) Hermite-Gaussian mode, its amplitude has a transversal $\pi$ phase slip which will be transferred to the atoms thus creating a soliton. Using this method it is possible to circumvent the restriction set by the diffraction limit. The method allows one to create multicomponent (vector) solitons of the dark-bright form as well as the dark-dark combination. In addition it is possible to create in a controllable way two or more slowly moving dark solitons close to each other for studying the collisional properties. For this the first beam $\Omega_{1}$ should represent a superposition of the zero and second order Hermite-Gaussian modes in the second stage. The soliton collisions will be considered in more detail elsewhere.

\section{Acknowledgements}

This work was supported by the Alexander-vonHumboldt Foundation through the institutional collaborative grant between the University of Kaiserslautern and the Institute of Theoretical Physics and Astronomy of Vilnius University. P. Ö. acknowledges support from the EPSRC and the Royal Society of Edinburgh.

\section{References}

[1] M.H. Anderson, J.R. Ensher, M.R. Matthews, C.E. Wieman, and E.A. Cornell, Science 269, 198 (1995).

[2] C.C. Bradley, C.A. Sackett, J.J. Tollett, and R.G. Hulet, Phys. Rev. Lett. 75, 1687 (1995).

[3] K.B. Davis, M.-O. Mewes, M.R. Andrews, N.J. van Druten, D.S. Durfee, D.M. Kurn, and W. Ketterle, Phys. Rev. Lett. 75, 3969 (1995).

[4] See, e. g., S. Stringari and L. Pitaevskii, Bose-Einstein Condensation (Clarendon Press, Oxford, 2003).

[5] Y.S. Kivshar and B. Luther-Davies, Phys. Rep. 298, 81 (1998).

[6] S. Burger, K. Bongs, S. Dettmer, W. Ertmer, K. Sengstock, A. Sanpera, G.V. Shlyapnikov, and M. Lewenstein, Phys. Rev. Lett. 83, 5198 (1999).

[7] J. Denschlag, J.E. Simsarian, D.L. Feder, C.W. Clark, L.A. Collins, J. Cubizolles, L. Deng, E.W. Hagley, K. Helmerson, W.P. Reinhardt, S.L. Rolston, B.I. Schneider, and W.D. Phillips, Science 287, 97 (2000).
[8] L. Khaykovich, F. Schreck, G. Ferrari, T. Bourdel, J. Cubizolles, L.D. Carr, Y. Castin, and C. Salomon, Science 296, 1290 (2002).

[9] K.E. Strecker, G.B. Partridge, A.G. Truscott, and R.G. Hulet, Nature 417, 150 (2002).

[10] A.E. Muryshev, H.B. van Linden van den Heuvell, and G.V. Shlyapnikov, Phys. Rev. A 60, R2665 (1999).

[11] Th. Busch and J.R. Anglin, Phys. Rev. Lett. 84, 2298 (2000).

[12] P. Öhberg and L. Santos, Phys. Rev. Lett. 86, 2918 (2001).

[13] Th. Busch and J.R. Anglin, Phys. Rev. Lett. 87, 010401 (2001).

[14] G.A. El, A. Gammal, and A.M. Kamchatnov, Phys. Rev. Lett. 97, 180405 (2006).

[15] W.P. Reinhardt and C.W. Clark, J. Phys. B 30, L785 (1997).

[16] T.F. Scott, R.J. Ballagh, and K. Burnett, J. Phys. B 31, L329 (1998).

[17] V.A. Brazhnyi and A.M. Kamchatnov, Phys. Rev. A 68 , 043614 (2003).

[18] Z. Dutton, M. Budde, C. Slowe, and L.V. Hau, Science 293, 663 (2001).

[19] N.S. Ginsberg, J. Brand, and L.V. Hau, Phys. Rev. Lett. 94, 040403 (2005).

[20] Ł. Dobrek, M. Gajda, M. Lewenstein, K. Sengstock, G. Birkl, and W. Ertmer, Phys. Rev. A 60, R3381 (1999).

[21] L.D. Carr, J. Brand, S. Burger, and A. Sanpera, Phys. Rev. A 63, 051601 (2001).

[22] B. Wu, J. Liu, and Q. Niu, Phys. Rev. Lett. 88, 034101 (2002).

[23] V.E. Zakharov and A.B. Shabat, Zh. Eksp. Teor. Fiz. 64, 1627 (1973) [Sov. Phys. JETP 37, 823 (1973)].

[24] S. Burger, L.D. Carr, P. Öhberg, K. Sengstock, and A. Sanpera, Phys. Rev. A 63, 043611 (2002).

[25] J. Ruseckas, G. Juzeliūnas, P. Öhberg, and M. Fleischhauer, Phys. Rev. Lett. 95, 010404 (2005).

[26] R.G. Unanyan, M. Fleischhauer, B.E. Shore, and K. Bergmann, Opt. Commun. 155, 144 (1998).

[27] K.P. Marzlin, W.P. Zhang, and E.M. Wright, Phys. Rev. Lett. 79, 4728 (1997).

[28] G. Nandi, R. Walser, and W.P. Schleich, Phys. Rev. A 69, 063606 (2004).

[29] M.F. Andersen, C. Ryu, P. Clade, V. Natarajan, A. Vaziri, K. Helmerson, and W.D. Phillips, Phys. Rev. Lett. 97, 170406 (2006).

[30] G. Juzeliūnas, P. Öhberg, J. Ruseckas, and A. Klein, Phys. Rev. A 71, 053614 (2005).

[31] G. Juzeliūnas, J. Ruseckas, and P. Öhberg, Lithuanian J. Phys. 45, 191 (2005).

[32] M.V. Berry, Proc. R. Soc. London, Sec. A 392, 45 (1984).

[33] C.A. Mead, Rev. Mod. Phys. 64, 51 (1992).

[34] F. Wilczek and A. Zee, Phys. Rev. Lett. 52, 2111 (1984). 


\title{
SOLITONU SUKŪRIMAS ATOMU BOZE் IR EINŠTEINO KONDENSATUOSE, ADIABATIŠKAI KEIČIANT TAMSIAS BŪSENAS
}

\author{
G. Juzeliūnas $^{\text {a }}$, J. Ruseckas ${ }^{\text {a }}$, P. Öhberg ${ }^{\mathrm{b}}$, M. Fleischhauer ${ }^{\mathrm{c}}$ \\ ${ }^{a}$ Vilniaus universiteto Teorines fizikos ir astronomijos institutas, Vilnius, Lietuva \\ ${ }^{\mathrm{b}}$ Heriot-Watt universitetas, Edinburgas, Jungtine karalystè \\ ${ }^{\mathrm{c}}$ Kaizerslauterno technikos universitetas, Kaizerslauternas, Vokietija
}

\section{Santrauka}

Pasiūlytas naujas būdas solitonams sukurti pailguose Bozè ir Einšteino kondensatuose. Solitonai sukuriami braukiant trimis lazerių pluoštais per kondensatą. Jei vienas iš pluoštų aprašomas pirmos eilès (TEM10) Ermito ir Gauso moda, elektrinio lauko amplitudè pluošto centre turi skersini $\pi$ dydžio fazès šuolị, kuris gali būti perkeltas atomams, taip sukuriant solitoną. Naudojant mūsų būdą, galima išvengti difrakcijos apribojimo fazès šuoliui, pasireiškiančio ịprastame solitonų sukūrimo metode, apšviečiant dali kondensato ir taip užrašant fazę. Mūsų metodas leidžia sukurti daugiakomponenčius (vektorinius) tamsaus-tamsaus bei tamsausšviesaus pavidalo solitonus. Be to, galima kontroliuojamai sukurti du ar daugiau lètai judančius solitonus, esančius arti vienas kito, ir taip tirti jų susidūrimus. 